The Astrophysical Journal, 299:L57-L61, 1985 December 1

C 1985. The American Astronomical Society. All rights reserved. Printed in U.S.A.

\title{
SOLAR CORONAL AND PHOTOSPHERIC ABUNDANCES FROM SOLAR ENERGETIC PARTICLE MEASUREMENTS
}

\author{
H. H. Breneman and E. C. Stone \\ California Institute of Technology \\ Received 1985 A ugust 12; accepted 1985 September 17
}

\begin{abstract}
We find that the ionic charge-to-mass ratio $(Q / M)$ is the principal organizing parameter for the fractionation of solar energetic particles (SEPs) by acceleration and propagation processes and for flare-to-flare variability. Unfractionated coronal abundances for 20 elements with $6 \leq Z \leq 30$ are derived by applying a single-parameter $Q / M$-dependent correction to the average SEP abundances, and unfractionated photospheric abundances are obtained by applying an additional correction based on first ionization potential.
\end{abstract}

Subject headings: cosmic rays: abundances - Sun: abundances - Sun: atmosphere - Sun: corona Sun: flares

\section{INTRODUCTION}

During large solar flares, a sample of the corona can be accelerated to energies in excess of $10^{6} \mathrm{eV}$. Analysis of the composition of these solar energetic particles (SEPs) can yield accurate coronal abundances provided that the systematic fractionation introduced by the acceleration and interplanetary propagation processes can be modeled. These processes produce a flare-to-flare variability of SEP composition about the average abundances that has been described as a monotonic function of $Z$, variable in magnitude from flare to flare (e.g., Cook et al. 1979; McGuire, von Rosenvinge, and McDonald 1979; Cook, Stone, and Vogt 1980, 1984; Mason et al. 1980; Meyer 1981, 1985). When compared with "solar system" or photospheric abundances, the average SEP abundances exhibit an additional fractionation which depends on first ionization potential (Hovestadt 1974; Webber 1975; Cook et al. 1979; McGuire, von Rosenvinge, and McDonald 1979; Meyer 1981, 1985; Cook, Stone, and Vogt 1984). In this Letter, new high-precision SEP elemental abundance data are used to accurately define the fractionation processes, allowing a determination of unfractionated coronal and photospheric abundances for 20 elements.

\section{RESULTS AND DISCUSSION}

The data were collected by the Cosmic Ray Subsystem (CRS) aboard the Voyager 1 and Voyager 2 spacecraft (Stone et al. 1977). Each CRS includes four Low Energy Telescopes (LET) and two High Energy Telescopes (HET) employing silicon solid-state detectors and covering a combined incident energy range for oxygen of $3.5-50 \mathrm{MeV}$ per nucleon. Solar energetic particle abundances were obtained from a combined set of 10 solar flares that occurred during the 1977-1982 time period. Even relatively rare elements such as $\mathrm{Ar}$ and $\mathrm{Ca}$ are clearly resolved, as shown in Figure 1. For the rarer elements (e.g., $\mathrm{P}, \mathrm{Cl}, \mathrm{K}$ ), the abundances were determined by performing maximum-likelihood fits to the rare element peak and its two usually more abundant neighbors. For the most abundant elements (C, N, O, Ne, $\mathrm{Na}, \mathrm{Mg}, \mathrm{Al}, \mathrm{Si}, \mathrm{S}, \mathrm{Ar}, \mathrm{Ca}, \mathrm{Cr}, \mathrm{Fe}$ ), the abundances could be determined in each of the flares; these values were averaged to obtain mean abundances using a weighting technique that takes into account both statistical variations and real abundance variations from flare to flare (Breneman 1985). The resulting average SEP abundances relative to silicon (Table 1) agree well with those obtained by other recent investigations (Mason et al. 1980; Cook, Stone, and Vogt 1984; Meyer 1985) for the more abundant elements but have about a factor of 3 higher precision. In addition, statistically significant abundances of several rarer elements are obtained for the first time.

Individual flares exhibit systematic abundance variations relative to the average abundances in Table 1 . Using measured SEP ionic charge states (Luhn et al. 1984) for C, N, O, Ne, $\mathrm{Mg}, \mathrm{Si}, \mathrm{S}$, and $\mathrm{Fe}$, and using the calculations of Shull and van Steenberg (1982) to interpolate for other elements, we find that the flare-to-flare variability exhibits a monotonic dependence on the ionic charge-to-mass ratio $Q / M$ of the particles, as illustrated in Figure 2. Such a dependence is not unexpected since acceleration and propagation processes depend on the rigidity of the ions which is inversely proportional to $Q / M$. Since individual flares exhibit a $Q / M$-dependent fractionation with respect to the average of all flares, there is also likely a residual $Q / M$-dependent fractionation of the average flare composition with respect to the corona.

Since the elements with low first ionization potential (FIP $<9 \mathrm{eV}$ ) display no FIP-dependent fractionation, the correction factor for the residual $Q / M$-dependent fractionation has been determined by comparing the average SEP abundances of these elements to photospheric spectroscopic abundances (Grevesse 1984) (see Fig. 3). The correction function, which is assumed to be a power law in $Q / M$, has a best-fit slope of $0.66 \pm 0.17$ and may be applied to the SEP abundances for all elements, resulting in unfractionated SEP-derived coronal abundances (Table 1). These abundances agree well with 


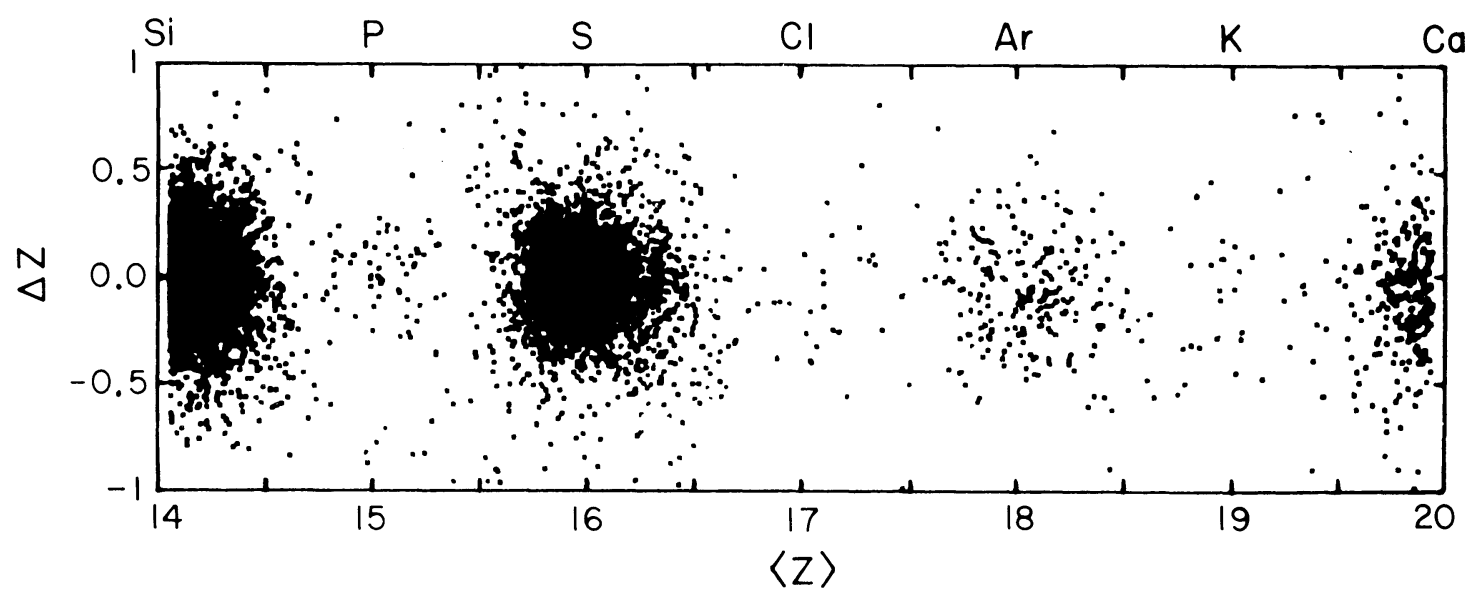

FIG. 1. - Scatterplot of SEP data from LET in the $Z=14-20$ charge range. $\langle Z\rangle$ is the average, and $\Delta Z$ the difference, of two essentially independent charge determinations obtained from the LET data for each analyzed particle.

TABLE 1

Solar Energetic Particle Average Elemental Abundances and Solar Energetic Particle-derived Coronal AND PhotosPheric ABUndances

\begin{tabular}{|c|c|c|c|c|}
\hline \multirow[b]{2}{*}{$Z$} & \multirow[b]{2}{*}{ ElEMENT } & \multicolumn{3}{|c|}{ ABUNDANCE $(\mathrm{Si}=1000)$} \\
\hline & & SEP & $\begin{array}{l}\text { SEP-derived } \\
\text { Corona }\end{array}$ & $\begin{array}{l}\text { SEP-derived } \\
\text { Photosphere }\end{array}$ \\
\hline $6 \ldots \ldots$ & $\mathrm{C}$ & $2710 .+240$ & $2350 .+230$ & {$\left[6490 .{ }_{-270}^{+280}\right]^{\mathrm{a}}$} \\
\hline $7 \ldots \ldots$ & $\mathrm{N}$ & 775. +54 & 700. +52 & {$[2775 .+53 .]_{-50}^{\mathrm{a}}$} \\
\hline $8 \ldots$ & $\mathrm{O}$ & $6230 .+340$ & $\begin{array}{l}5680 .+360 \\
-340\end{array}$ & {$[22900 .]^{\mathrm{a}}$} \\
\hline $9 \ldots$ & $\mathrm{F}$ & $\left(0.30_{-0.30}^{+0.32}\right)^{\mathrm{b}}$ & $\left(0.28_{-0.28}^{+0.29}\right)^{\mathrm{b}}$ & {$\left[\left(1.1_{-1.1}^{+1.2}\right)^{\mathrm{b}}\right]^{\mathrm{a}}$} \\
\hline $10 \ldots$ & $\mathrm{Ne}$ & 887. ${ }_{-83}^{+91}$ & 783. +84 & {$\left[3140 .{ }_{-195}^{+205}\right]^{\mathrm{a}}$} \\
\hline $11 \ldots$ & $\mathrm{Na}$ & $73.3_{-6.6}^{+7.2}$ & $67.0_{-6.2}^{+6.8}$ & $\begin{array}{l}67.0_{-6.2}^{+6.8} \\
{ }^{+6}\end{array}$ \\
\hline $12 .$. & $\mathrm{Mg}$ & 1206. ${ }_{-61}^{+64.0}$ & 1089. ${ }_{-62 .}^{+64.2}$ & $\begin{array}{l}\text { 1089. } \\
+64 . \\
62 .\end{array}$ \\
\hline $13 \ldots$ & $\mathrm{Al}$ & $87.4_{-4.1}^{+4.3}$ & $83.7_{-4.0}^{+4.2}$ & $83.7_{-4.0}^{+4.2}$ \\
\hline $14 \ldots \ldots$ & $\mathrm{Si}$ & 1000 & 1000 & 1000 \\
\hline $15 \ldots \ldots$ & $\mathrm{P}$ & $4.61_{-0.67}^{+0.62}$ & $4.89_{-0.72}^{+0.66}$ & {$\left[9.24_{-1.54}^{+1.46}\right]^{\mathrm{a}}$} \\
\hline $16 \ldots \ldots$ & $\mathrm{S}$ & $222+8$ & $242 .+10$ & {$[460 .]^{\mathrm{a}}$} \\
\hline $17 \ldots \ldots$ & $\mathrm{Cl}$ & $2.05_{-0.68}^{+0.72}$ & $2.38_{-0.80}^{+0.84}$ & {$\left[9.6_{-3.3}^{+3.5}\right]^{\mathrm{a}}$} \\
\hline $18 \ldots \ldots$ & $\mathrm{Ar}$ & $20.7_{-3.0}^{+3.5}$ & $24.1_{-3.6}^{+4.2}$ & {$[102 .+20 \cdot]_{-17}^{\mathrm{a}}$} \\
\hline $19 \ldots \ldots$ & $\mathrm{K}$ & $3.3_{-1.3}^{+1.7}$ & $3.9_{-1.6}^{+2.0}$ & $3.9_{-1.6}^{+2.1}$ \\
\hline $20 \ldots \ldots$ & $\mathrm{Ca}$ & $68 ._{-10}^{+12 .}$ & $82 .{ }_{-12}^{+14.0}$ & $82 .{ }_{-12}^{+14.6}$ \\
\hline $21 \ldots \ldots$ & $\mathrm{Sc}$ & $\left(0.24_{-0.24}^{+0.44}\right)^{\mathrm{b}}$ & $\left(0.31_{-0.31}^{+0.55}\right)^{\mathrm{b}}$ & $\left(0.31_{-0.31}^{+0.55}\right)^{\mathrm{b}}$ \\
\hline 22 . & $\mathrm{Ti}$ & $3.8_{-1.0}^{+1.2}$ & $4.9_{-1.3}^{+1.6}$ & $4.9_{-1.3}^{+1.6}$ \\
\hline $23 .$. & $\mathrm{V}$ & $\left(0.37_{-0.37}^{+0.53}\right)^{\mathrm{b}}$ & $\left(0.48_{-0.48}^{+0.69}\right)^{\mathrm{b}}$ & $\left(0.48_{-0.48}^{+0.69}\right)^{\mathrm{b}}$ \\
\hline $24 \ldots \ldots$ & $\mathrm{Cr}$ & $14.3_{-2.4}^{+2.9}$ & $18.3_{-3.3}^{+3.9}$ & $18.3_{-3.3}^{+3.9}$ \\
\hline $25 \ldots \ldots$ & $\mathrm{Mn}$ & $\begin{array}{l}5.2_{-2.0}^{+3.0} \\
\end{array}$ & $\begin{array}{r}6.8_{-2.7}^{+3.9} \\
\end{array}$ & $\begin{array}{r}6.8_{-2.7}^{+3.9} \\
\end{array}$ \\
\hline $26 \ldots \ldots$ & $\mathrm{Fe}$ & 959. +.94 & $1270 ._{-150}^{+170}$ & $\begin{array}{c}1270 .+170 \\
-150\end{array}$ \\
\hline $27 \ldots \ldots$ & Co & $<13.2$ & $<18.1$ & $<18.1$ \\
\hline $28 \ldots \ldots$ & $\mathrm{Ni}$ & $33.8_{-4.7}^{+5.2}$ & $46.5_{-7.4}^{+8.1}$ & $46.5_{-7.4}^{+8.1}$ \\
\hline $29 \ldots \ldots$ & $\mathrm{Cu}$ & $\left(0.39_{-0.39}^{+0.59}\right)^{\mathrm{b}}$ & $\left(0.57_{-0.57}^{+0.87}\right)^{\mathrm{b}}$ & $\left(0.57_{-0.57}^{+0.87}\right)^{\mathrm{b}}$ \\
\hline $30 \ldots \ldots$ & $\mathrm{Zn}$ & $1.06_{-0.49}^{+0.56}$ & $1.61_{-0.76}^{+0.87}$ & $1.61_{-0.76}^{+0.87}$ \\
\hline
\end{tabular}

${ }^{a} \mathrm{~N}, \mathrm{~F}, \mathrm{Ne}, \mathrm{Cl}$, and $\mathrm{Ar}$ have been renormalized to oxygen. The stated uncertainties are relative to oxygen and do not include a systematic uncertainty of $\sim 6 \%$ due to the uncertainty in the FIP fractionation which is assumed to be the same as for oxygen. Similarly, $\mathrm{P}$ is renormalized to $\mathrm{S}$ and both have a systematic uncertainty of $\sim 9 \%$ from the sulfur fractionation correction. The $\mathrm{C}$ fractionation uncertainty is assumed to be a factor of 1.5 in order to include the fractionation values for $\mathrm{O}$ and $\mathrm{S}$. The illustrative calculation by Geiss and Bochsler 1984 suggests that the precise ionization fractionation depends on several parameters and may differ by $\sim 30 \%$ from those assumed.

${ }^{b}$ Abundances for these elements are based on five or fewer particles and are highly uncertain. 


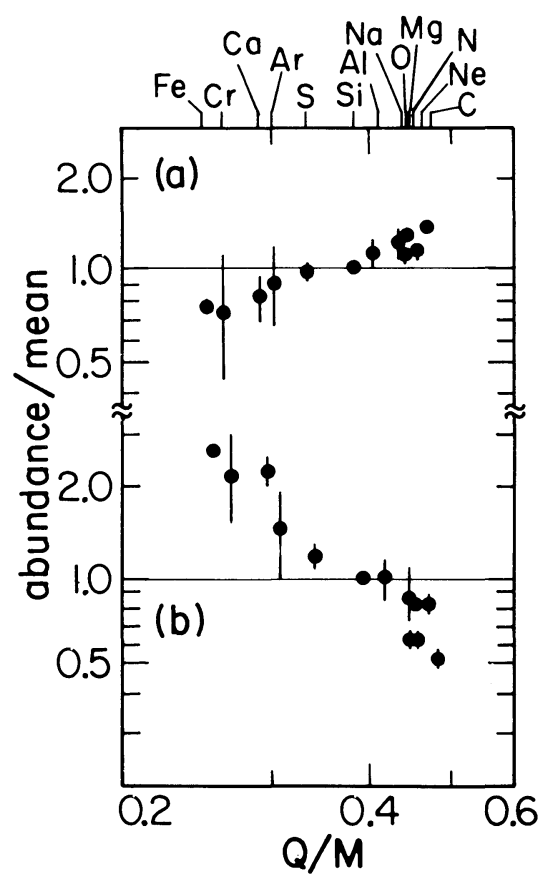

FIG. 2.-Abundances relative to the mean SEP abundance for two typical flares, plotted vs. $Q / M$. The data were collected during the time periods ( $a$ ) 1978 April $21-29$ and $(b) 1977$ September $24-27$. The $Q / M$-dependent abundance fractionation can be represented by $(Q / M)^{\alpha}$.

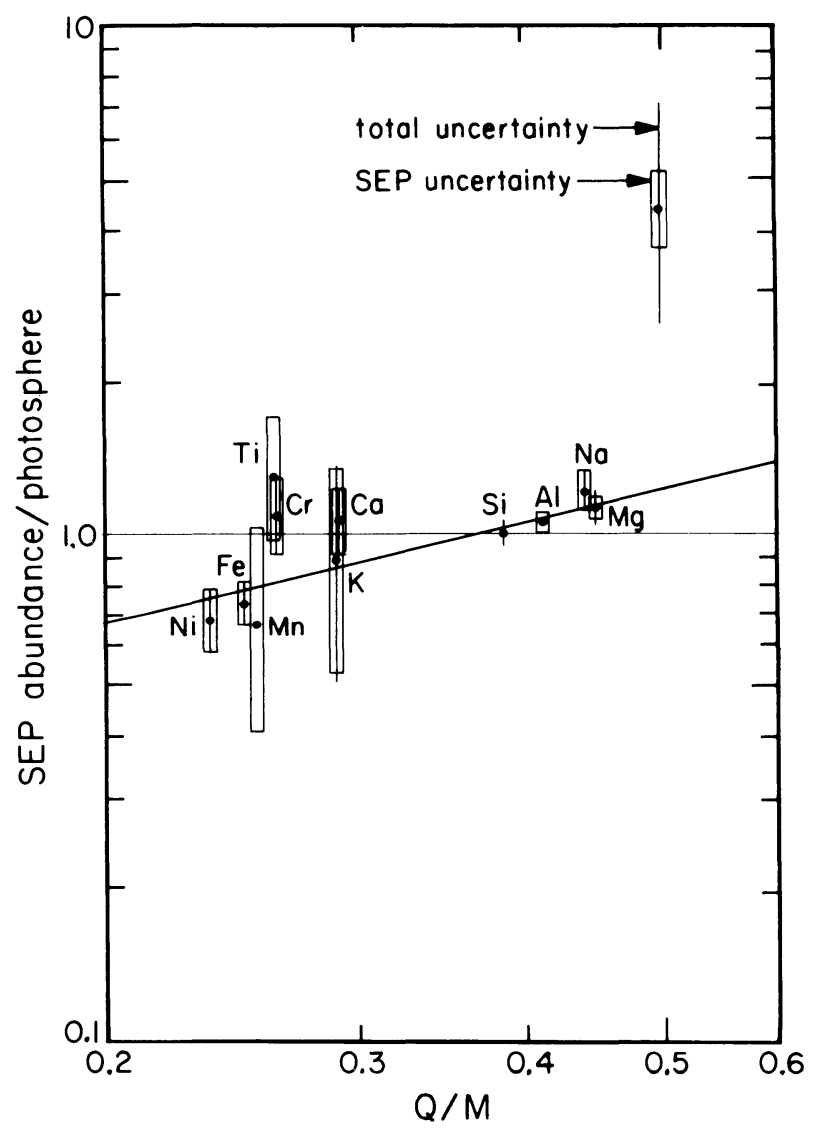

FIG. 3.- Mean SEP abundance relative to the photosphere (Grevesse 1984) for the low-FIP elements, plotted vs. $Q / M$. The total uncertainty is indicated for all elements for which there is a quoted uncertainty in the spectroscopically derived photospheric abundances. The fractionation of the SEP abundances relative to the photosphere is represented by a power law in $Q / M$ which is fitted to all of the elements shown except $\mathrm{Al}$, Ti, and $\mathrm{Mn}$, which have unknown photospheric uncertainties. The reduced $\chi^{2}$ of the fit is 1.0 . 


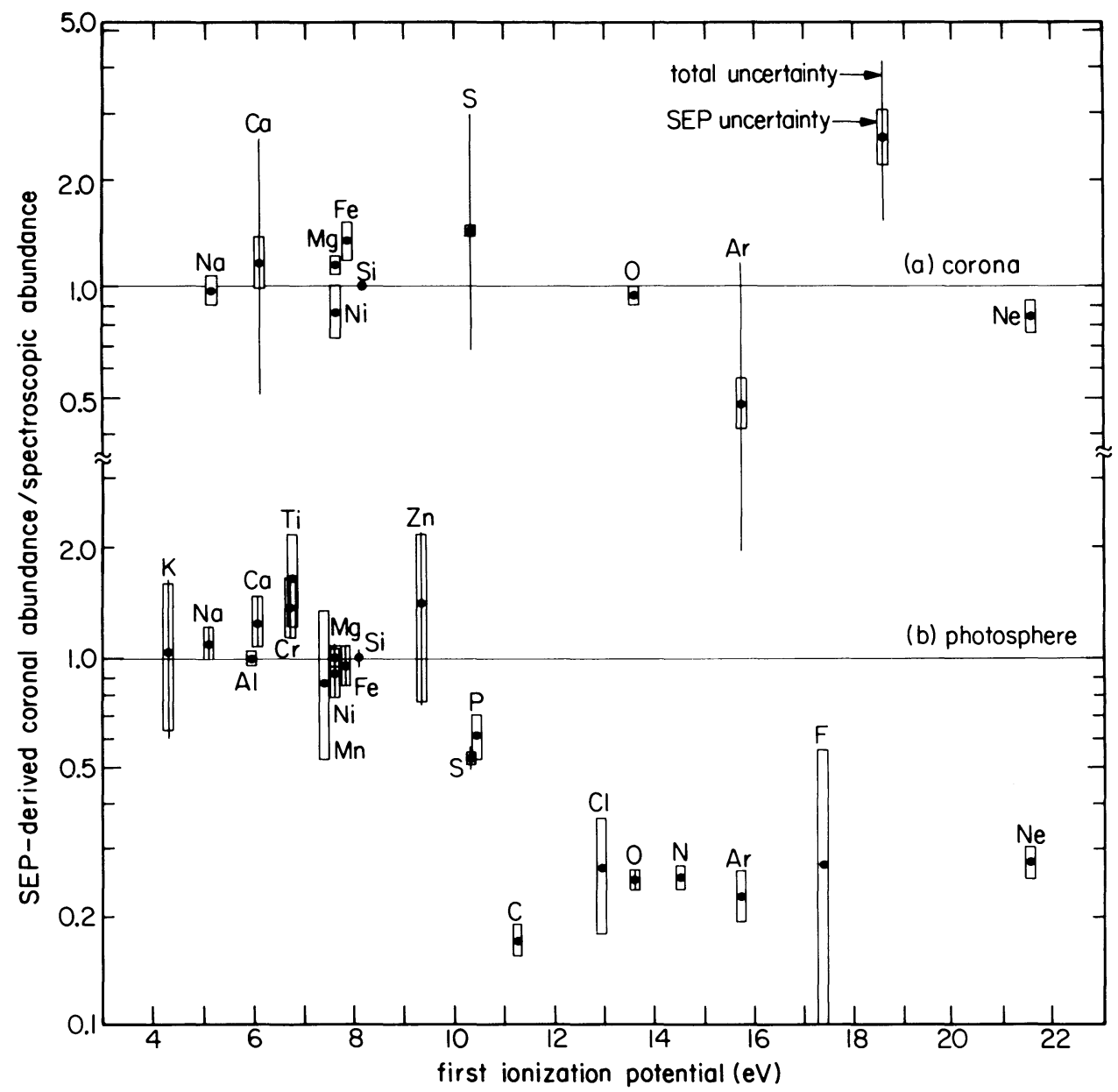

FIG. 4. - (a) SEP-derived coronal abundances relative to spectroscopically derived coronal abundances (Veck and Parkinson 1981), plotted vs. FIP. Spectroscopic uncertainties are quoted only for S, Ar, and Ca. (b) SEP-derived coronal abundances relative to spectroscopic photospheric abundances (Grevesse 1984), plotted vs. FIP. Total uncertainties are shown for only those elements with quoted uncertainties in the photospheric abundance. Unlike the majority of elements, the photospheric abundances of $\mathrm{C}, \mathrm{N}, \mathrm{O}, \mathrm{F}$, and $\mathrm{Cl}$ were obtained primarily from molecular spectral lines, while those of Ne and Ar were inferred from other solar and stellar abundance data.

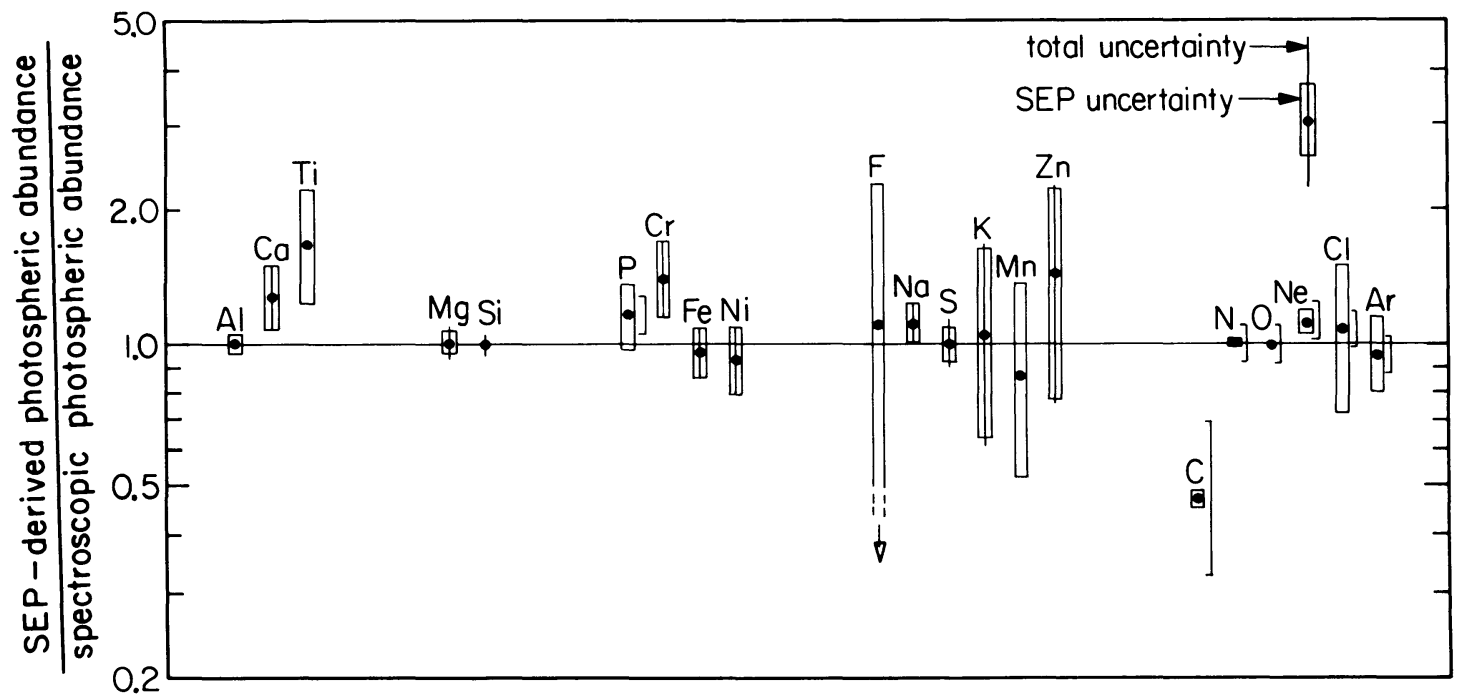

FIG. 5.-SEP-derived photospheric abundances relative to spectroscopically derived photospheric abundances (Grevesse 1984). Total uncertainties are shown for only those elements with quoted uncertainties in the spectroscopically derived abundances. The estimated uncertainties in the FIP fractionation correction are separately indicated by the vertical brackets. 
coronal abundances obtained by XUV/X-ray spectroscopy (see Fig. $4 a$ ), but have much higher precision and are available for many more elements.

In Figure $4 b$, the SEP-derived coronal abundances are compared to the Grevesse (1984) photospheric abundances; this shows more clearly the step-function nature of the FIPdependent fractionation previously reported (Cook et al. 1979; Meyer 1981, 1985; Cook, Stone, and Vogt 1984). The dynamical ionization model of Geiss and Bochsler (1984) for the formation of the corona from photospheric material suggests that high-FIP elements such as N, O, F, Ne, Cl, and Ar, which cannot be ionized from the ground state by the large flux of $\mathrm{H}$ Ly $\alpha$ photons, are depleted because their ionization times are longer than the time individual atoms spend in a rising spicule. Since this model indicates that the ionized fractions of $\mathrm{N}, \mathrm{O}, \mathrm{Ne}$, and $\mathrm{Ar}$ are similar, their abundances are corrected by the depletion factor of oxygen in SEPs relative to the photosphere $(4.03 \pm 0.26)$. In the absence of detailed calculations, the abundances of $\mathrm{F}$ and $\mathrm{Cl}$, which have similar FIPs, are corrected by this same factor, while $\mathrm{P}$ and $\mathrm{S}$ have lower
FIPs and are corrected by the depletion factor of sulfur $(1.89 \pm 0.17)$, and $\mathrm{C}$, having an intermediate FIP, is corrected by the geometric mean of the oxygen and sulfur depletion factors. The low-FIP elements, which are quickly ionized, should be unfractionated and therefore require no correction.

The resulting SEP-derived photospheric abundances (Table 1) involve different modeling parameters than spectroscopic determinations and are available for some elements (e.g., $\mathrm{C}, \mathrm{N}, \mathrm{Ne}, \mathrm{Ar}$ ) that are difficult or impossible to observe spectroscopically. The main differences (Fig. 5) are a significantly higher abundance of $\mathrm{Cr}$ (and possibly $\mathrm{Ca}$ and $\mathrm{Ti}$ ) and a $\mathrm{C}$ abundance that is about half of the commonly assumed solar abundance. The SEP-derived $\mathrm{Fe} / \mathrm{Si}$ ratio is in agreement with the recent photospheric value (Grevesse 1984), which is 50\% larger than the meteoritic value.

We appreciate the contributions of R. E. Vogt and other CRS team members. This work has been supported in part by NASA under contract NAS7-918 and grant NGR 05-002-160.

\section{REFERENCES}

Breneman, H. H. 1985, Ph.D. thesis, California Institute of Technology. Cook, W. R., Stone, E. C., and Vogt, R. E. 1980, Ap. J. (Letters), 238 L97. 1984, Ap. J., 279, 827.

Cook, W. R., Stone, E. C., Vogt, R. E., Trainor, J. H., and Webber, W. R. 1979, in Proc. 16th Int. Cosmic Ray Conf. (Kyoto), 12, 265.

Geiss, J., and Bochsler, P. 1984, in Proc. Int. Conf. on Isotopic Ratios in the Solar System (Paris), in press.

Grevesse, N. 1984, Phys. Scripta, T8, 49.

Hovestadt, D. 1974, in Solar Wind III, ed. C. T. Russell (Los Angeles: Institute of Geophysics and Planetary Physics, University of California), p. 2.

Luhn, A., Klecker, B., Hovestadt, D., Gloeckler, G., Ipavich, F. M., Scholer, M., Fan, C. Y., and Fisk, L. A. 1984, Adv. Space Res., 4, No. $2-3,161$.

Mason, G. M., Fisk, L. A., Hovestadt, D., and Gloeckler, G. 1980, Ap. J., 239, 1070 .

McGuire, R. E., von Rosenvinge, T. T., and McDonald, F. B. 1979, in Proc. 16th Int. Cosmic Ray Conf. (Kyoto), 5, 61.

Meyer, J.-P. 1981, in Proc. 17th Int. Cosmic Ray Conf. (Paris), 3, 145. 1985, Ap. J. Suppl., 57, 151.

Shull, J. M., and van Steenberg, M. 1982, Ap. J. Suppl., 48, 95

Stone, E. C., Vogt, R. E., McDonald, F. B., Teegarden, B. J., Trainor, J. H. Jokipii, J. R. and Webber, W. R. 1977, Space Sci. Rev. 21, 355. Veck, N. J., and Parkinson, J. H. 1981, M.N.R.A.S., 197, 41.

Webber, W. R. 1975, in Proc. 14th Int. Cosmic Ray Conf. (Munich), 5, 1597.

H. H. Breneman and E. C. Stone: 220-47 Downs Laboratory, California Institute of Technology, Pasadena, CA 91125 